\title{
OBSERVATIONS ON QUESTIONNAIRE CONCERNING CONGENITAL SYPHILIS
}

\author{
By DAVID LEES, F.R.C.S.
}

THE series of questions which have been circulated by the President cover a wide field and raise many controversial issues. In our opinion mercury should not be relied on alone as a method of treatment, whether administered orally, by inunction, or intramuscularly. Daily inunction is of value in very young, emaciated and debilitated children, but it should be used as an adjuvant to the arsenicals and not as the main method of attack on the syphilitic infection. Occasionally, if there are digestive disturbances and green stools, in the case of these children, hydrarg cum creta may be given with good results, but more as an intestinal antiseptic than as an anti-syphilitic drug. In our experience inunction is very perfunctorily carried out by most mothers, and the placing of a little mercury ointment under a child's binder is a travesty of inunction.

Arsenobenzol derivatives may be administered with complete safety to most children from birth onwards, and is the best single form of treatment. We use almost exclusively the intramuscular method, the dose being regulated by the weight of the child from week to week. It is preferable to begin with small doses bi-weekly rather than with a larger dose once weekly, and progressive increase of weight is the best indication of tolerance and of the correct dosage. Allowing I centigramme of " 9 I 4 " per kilo of bodyweight, we give an initial dose of about half of this in the first week and successive doses are increased according to tolerance. At the end of the first ten days' treatment bismuth may also be administered in conjunction with the arsenobenzol drug. As in acquired syphilis the combination of drugs is better than either alone. The dose of bismuth should also be adjusted according to the weight of the child. The " 9I4" preparations which we prefer are kharsulphan, myosalvarsan, and in some of the very young children 


\section{BRITISH JOURNAL OF VENEREAL DISEASES}

we have recently been using acetylarsan with very good results. Of the bismuth preparations we prefer bismogenol, or bismuth oxychloride in infancy, and if fairly rapid absorption is required. Later in the course of the treatment, metallic bismuth is given in the form of bismuth hypoloid or bismostab. Stabismol, bivatol and neo-cardyl are also used if more rapid absorption still is indicated. They are not required except in cases which cannot tolerate injections of "9I4," and, when the latter is given concurrently, rapid absorption of the bismuth preparation is not required for any rapid spirillicidal effect.

We prefer to treat most cases with arsenobenzol and bismuth for a period of eight to ten weeks, and follow this with a period of three to four weeks rest to allow for elimination of the drugs. During this rest period and often also throughout the course, syrup of iodide of iron is administered in conjunction with general tonics such as cod liver oil, numol, radio-malt, etc.

In older children, three to fourteen years old, syrup of iodide of iron or some other form of iron such as ferri et ammon. cit., is often given continuously as a general tonic over long periods. The adjuvant treatment is largely administered for the relief of concomitant conditions, e.g., iron in various forms in anæmia, cod liver oil and vitamins in rickety children. Successive courses are given every three months and the average period of observation is three to four years.

Toxic effects from treatment by arsenobenzol, bismuth and iodides are rarely met with, and are the exception.

No cases of dermatitis have occurred in a ten-year period during which over I,O0O cases of congenital syphilis have been treated by us. One case of jaundice occurred, but it was very mild and arsenobenzol injections were resumed three months later.

Occasionally there is a case of transient albuminuria. We have had one case of purpura.

Stomatitis, or any other form of intolerance from bismuth injections or injections of mercury has been unknown. During this ten-year period a total of over 60,000 injections of various metals have been administered to these patients with practically no serious effects and with marked clinical improvement.

We consider that interstitial keratitis in the child 


\section{TREATMENT OF CONGENITAL SYPHILIS}

should be treated just as it is treated when it eventuates in adult-acquired syphilis, by iodides, arsenobenzol, and bismuth. It is important to hospitalise acute cases and to keep the pupil under the influence of atropine till the acute symptoms subside. Initially small and frequently repeated doses of "9I4" are preferable. Active treatment along these lines often obviates the second eye being involved and is the best method of preventing relapses. In our experience these relapses are often the result of inadequate treatment or of treatment over an insufficient period of time.

Juvenile neurosyphilis occurs more frequently than is generally recognised. It is often very asymptomatic and only detectable by examination of the cerebrospinal fluid.

Our results in treating juvenile neurosyphilis with intravenous injections of tryparsamide combined with intramuscular injections of bismuth have been exceedingly good, both in the relief of any clinical symptoms, in the improvement of the child's general health, and in the sterilisation of the blood and cerebrospinal fluid. Vascular and meningo-vascular cases give the most favourable results, but good results are also obtained in juvenile tabes and paresis.

In juvenile G.P.I. malaria therapy has given us favourable results in approximately 29 per cent. of cases, an almost parallel figure to the results obtained in G.P.I. following acquired syphilis. We consider it imperative to follow up the fever treatment by injections of tryparsamide and bismuth. We have not had experience of amblyopia in a congenital syphilitic patient following on the use of tryparsamide, even in those who, prior to treatment, showed signs of optic atrophy.

The question of whether children should be treated who are apparently healthy, whose W.R. is negative, but who are born of syphilitic patients, is most difficult. Everything depends on the adequacy of the treatment of the mother during the ante-natal period. All those children should certainly be kept under constant clinical observation and the Wassermann or other test should be done at the end of one month and possibly also three to six months later. If there is any doubt as to the adequacy of the mother's treatment, e.g., if commenced only at the seventh or eighth month of pregnancy, we consider it a safe precaution to treat the child post-natally, at the same 


\section{BRITISH JOURNAL OF VENEREAL DISEASES}

time as the mother is being treated. No dogmatic answer can be given regarding this, and every case must be considered on its merits and in the light of the following facts, that anti-syphilitic treatment in a child is a comparatively safe procedure, that failure to give it to a latent syphilitic may have serious consequences, such as iritis and keratitis, and that in the great majority of children the treatment acts as an invaluable tonic and materially benefits the child's health. 